\title{
Comparison of monoptic and dichoptic masking by light
}

\author{
MARC GREEN \\ Indiana University, Bloomington, Indiana \\ and \\ J. VERNON ODOM \\ University of West Virginia, Morgantown, West Virginia
}

\begin{abstract}
Observers detected drifting gratings presented on a steady uniform field or on a background of uniform flicker with the same time-averaged luminance. Masking effects were obtained whether the flicker mask was presented to the eye on the same side as the test grating or to the one on the opposite side. Unlike masking-by-light studies, in which the mask changes the mean luminance of the display, dichoptic effects were only slightly smaller than those obtained monoptically. Moreover, when observers detected flicker masked by gratings, the dichoptic effect was as large as or larger than the monoptic masking. These results are consistent with the view that masking by light involves separate steady-state luminance and transient components. It is likely that only the transient effect occurs under conditions of dichoptic stimulation.
\end{abstract}

'Kahneman (1968) has suggested that visual masking situations can be broken into two categories. "Masking by light" occurs when a target is presented in close spatial and temporal proximity to a background consisting of a uniform flash of light. "Masking by pattern" occurs when the background contains internal contours.

Kahneman and, subsequently, others (e.g., Turvey, 1973) have suggested that one of the properties that distinguish these varieties of masking is the relative magnitude of monoptic and dichoptic effects. It is suggested that masking by pattern can be strong when the mask is presented to one eye while the target is presented to the other. Conversely, masking by light is presumed to be very small or entirely absent when mask and target are viewed dichoptically.

The purpose of this article is to present an explanation for the apparent lack of dichoptic effect obtained with masking by light. We base our ideas on a model proposed by several authors (e.g., Baker, 1963; Sperling, 1965), who have concluded that masking by uniform flash can actually be broken down into two separate processes. One is a transient effect at the onset and offset of the mask. The other is a steady-state response to the increased luminance produced by the flash. In a previous paper (Green, 1981b), evidence was found to support a two-process model.

While undertaking this research, J.V.O. was supported by NIH Grant EY 04806. M. Green's mailing address is: Visual Science Department, Indiana University, Bloomington, IN 47405 . J. V. Odom's mailing address is: Department of Ophthalmology, School of Medicine, University of West Virginia, Morgantown, WV 26506.
Observers detected a low or a high spatial frequency grating masked by a 700 -msec conditioning flash. Transient masking occurred at conditioning flash on and offset only with the low spatial frequency test grating. On the other hand, a steady-state change of threshold occurred from both gratings for the duration of the flash. This result indicates that low and high spatial frequencies are differentially susceptible to the transient component of masking but respond similarly to the steady-state component. One possible reason for the difference between the size of the effect in monoptic and dichoptic masking by light is that the transient component occurs at a central level of the visual system but the steady-state adaptive masking is more peripheral. It is well established that light-adaptive effects are primarily monocular (Levelt, 1965/1968). For example, Blake, Breitmeyer, and Green (1980) found that the luminance of a uniform field seen with one eye had no effect on contrast sensitivity for gratings viewed with the other.

This suggests that if there were no change in timeaverage luminance during masking, monoptic and dichoptic masking should be of more similar size. This can be tested by using masked and unmasked conditions of the same time-average luminance. In our unmasked condition, the targets are presented on a uniform background of fixed mean luminance. The mask consisted of light-dark sinusoidal flicker, which averaged out to the same mean luminance as the uniform background. This created a transient mask of uniform flashes but without a change in timeaveraged luminance level. In the study reported below, monoptic and dichoptic flicker masking were compared. 


\section{METHOD}

\section{Observer}

Two observers served during the experiment. Both had normal vision when wearing corrective spectacles, and both were experienced in psychophysical judgments.

\section{Apparatus and Procedure}

Stimuli were presented on the faces of two Tektronix $5103 \mathrm{~N}$ oscilloscopes by means of the standard television technique. The observers viewed the screens dichoptically, each eye seeing a different CRT with the aid of a mirror haplescope. Fusion was aided by the presence of a small circle ( $15 \mathrm{~min}$ diam, $1.2 \mathrm{~min}$ width) located in the center of each screen, as well as by the dark surround. When fused, the visual display was seen as a single 8-deg-diam circular field that had a mean luminance of $4 \mathrm{~cd} / \mathrm{m}^{2}$. The stimuli consisted of 0.67 cycles/deg sine-wave gratings that drifted rightward at $8 \mathrm{~Hz}$ and/or 8- $\mathrm{Hz}$ sinusoidal flicker and the uniform field. The space and time-averaged luminance of the display was constant during the experiment to maintain a constant state of light adaptation, and artificial pupils were not employed.

At the beginning of each session, the observer placed his/her head on a chinrest, fused the two screens, and adapted for $5 \mathrm{~min}$ to the unmodulated raster. The observer detected the target in a two-alternative forced-choice procedure. Each trial consisted of a pair of 2 -sec observation intervals (marked by tones) separated by $1 \mathrm{sec}$. The target was presented during one of the intervals, and the observer's task was to indicate which of the two observation intervals contained the target. The thresholds were determined by a staircase procedure in which three correct responses in a row resulted in a $0.1-\log$ decrement in target contrast (when detecting gratings) or depth of modulation (when detecting flicker). An error at any time produced a similar size increment. By averaging the peaks and valleys of the staircase, it was possible to obtain a detection threshold corresponding to the $79.6 \%$ point on a psychometric function (Wetherill \& Levitt, 1965). In the masking condition, the procedure was repeated, except that a background appeared in both observation intervals. Estimated standard errors generally fell between 0.45 and $.55 \mathrm{~dB}$.

Four conditions (two monoptic and two dichoptic) were employed in the study. In both the monoptic and dichoptic conditions, observers detected (1) gratings masked by flicker and (2) flicker masked by gratings. In all cases, amplitude of the mask was varied from its own detection threshold $0.3 \mathrm{log}$ unit steps.

\section{RESULTS}

Figure 1 shows the effects of uniform flicker on detection of drifting test gratings. The masking effect is expressed as log threshold elevation, which is the $\log$ of the ratio of unmasked sensitivity divided by masked sensitivity. Mask amplitude is expressed in threshold units, the value 1 indicates the modulation depth needed to detect flicker, while higher numbers represent multiples of this value. In both monoptic and dichoptic stimulation, flicker produced little or no masking at low amplitudes. Once a critical level (4 to 8 times threshold) was reached, masking increased as a power function of flicker amplitude. For Observer C.S., monoptic stimulation produced a masking effect of about $0.2 \mathrm{log}$ units (a factor of .58) greater than that obtained in the dichoptic condition. The threshold elevations obtained from $\mathrm{Ob}-$ server L. J. were similar for monoptic and dichoptic stimulation.
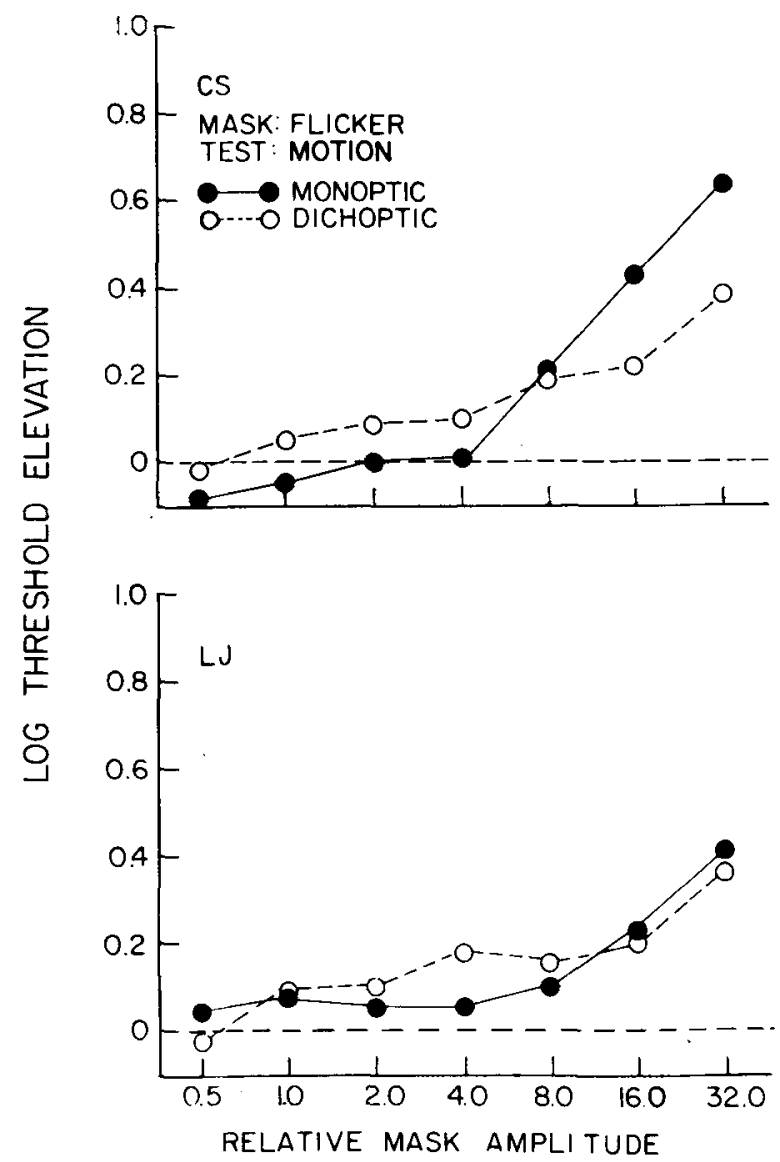

Figure 1. Log threshold elevation for detection of a drifting grating masked by uniform flicker. Filled symbols denote monoptic stimulation, and open symbols show data obtained by dichoptic presentation. In most cases, the masking effect reaches the OS confidence when threshold elevation is $.06 \log$ unit.

Results from a reverse experiment, detection of uniform flicker masked by gratings, are shown in Figure 2. For both observers, threshold elevation began at 2 times threshold and increased as a power function of the contrast of the masking grating. In the reverse experiment, however, dichoptic masking was as great as or greater than monoptic masking.

When the mask was at the 0.5 level, it was subthreshold and not visible to the observers. There is some indication that the subthreshold background may actually facilitate detection (C.S. detecting motion, and L.J. detecting flicker). Due to the small size of the effect and to the variability of the data, however, it is not clear if the facilitation effect is real. Therefore, we conducted an additional experiment with Observer L.J. The test stimulus was set at a fixed level of detectability, and the background (mask) was given one of four levels, 1.0 (its own detection level) $0.75,0.50$, or 0 . One hundred forced-choice trials were run at each background level. The results, shown in Figure 3, top, demonstrate that a sub- 


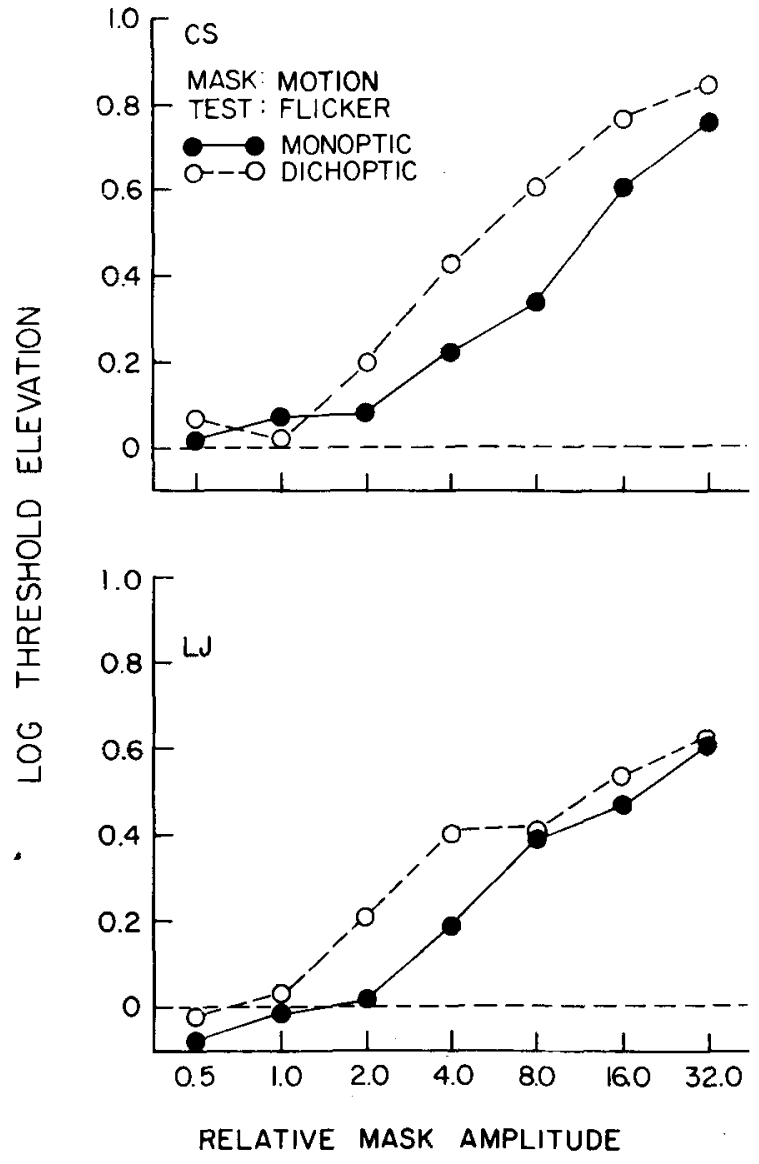

Figure 2. Log threshold elevation for detection of unfform ficker masked by a drifting ganting. Detrils are the same as in Figure 1.

threshold moving grating can facilitate detection of uniform flicker. The bottom panel shows the results obtained for two different contrasts of test grating. There is no clear indication that subthreshold flicker enhanced the detectability of test gratings. Similar results have been reported by Gorea (1979), who used orthogonal gratings as test and background.

\section{DISCUSSION}

The main finding of the present experiment was that when the steady-state luminance component is removed, monoptic masking and dichoptic masking by light differ very little in magnitude. At the highest masking levels, one observer exhibited similar-size monoptic and dichoptic effects, but the other showed a $0.2 \mathrm{log}$ unit monoptic superiority. These results contrast markedly with data obtained when the luminance component is present. For example, Wagman and Battersby (1959) found that the difference between monoptic and dichoptic masking was $2.5 \mathrm{log}$ units, whereas Boynton (1961) reported the results of two experiments in which the difference was 0.83 and $1.6 \log$ units. Our results are consistent with the view that the masking by light effect can be decomposed into transient and luminance components and that only the transient effect exhibits much strength under dichoptic conditions.

Although we obtained moderate-size dichoptic masking, other studies (e.g., Schiller, 1965) have failed to find any evidence for dichoptic masking by light. We believe that it might be possible to resolve the discrepancy on the basis of a sustained-transient model of masking by light. Several authors (e.g., Breitmeyer and Ganz, 1977; Tolhurst, 1973) have proposed that the human visual system contains separate "transient" mechanisms for perception of temporal change and "sustained" mechanisms for mediation of pattern vision. It has been proposed (Green, 1981b, 1983a) that the luminance component of masking affects both sustained and transient mechanisms but that the on-off mask transients desensitize only the transient system. This is suggested by the fact that

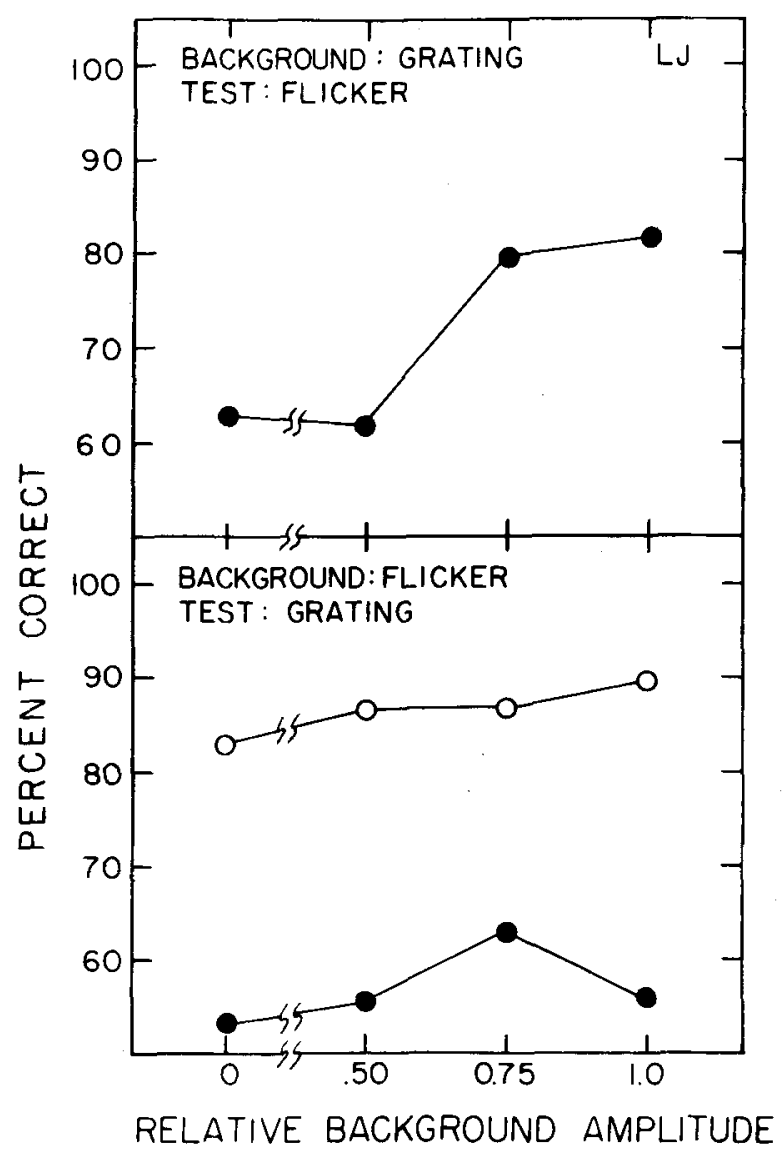

Percent correct for detection as a function of the amplitude of a subthreshold background. Top panel shows results obtained for detection of filcker on a grating background. Bottom panets show percent correct for detection of a grating on a ficker background. Open and closed symbols indicate two different contrast levels of the test grating. 
uniform flicker masks low-spatial-frequency moving gratings but affects neither high-spatial-frequency moving targets nor stationary gratings of any spatial frequency (Green, 1981a, 1983c, 1983a, 1983b). When target detection is mediated by the transient system, monocular masking should be large (both luminance and transient effects) and dichoptic masking should be moderate (the transient effect alone, since the luminance one does not transfer). If the sustained system mediates detection, then the monoptic effect should be large (luminance effect) but dichoptic masking should be absent (neither luminance nor transient effects). Therefore, dichoptic masking should occur only when detection is mediated by the transient system. Since the transient system is most sensitive at low spatial frequencies, this would be the case when targets were coarse gratings or spots of light. When the observer's task required pattern discrimination, however, no interocular transfer would occur. Schiller (1965) may have failed to find any dichoptic masking because he used a letter identification task, which presumably requires pattern vision mediated by the sustained system.

One of the surprising results of our study is that dichoptic masking could be larger than monoptic masking. Both observers exhibited this tendency when detection of flicker was masked by gratings. Legge (1979) has similarly reported a superiority of dichoptic masking in a study in which observers detected stationary gratings masked by other gratings. These results contrast with those of studies by subthreshold summation (e.g., Campbell \& Green, 1965; Green \& Blake, 1981; Matin, 1962) and orientation masking (Gilinsky \& Doherty, 1969), which generally find that dichoptic interactions are weaker than monoptic ones. Legge (1979) posited, and then rejected, the possibility that the larger dichoptic effect might be due to binocular suppression. His conclusion was based on the view that suppression and masking were mediated by different mechanisms. For example, when both eyes are used, masking is greatest when the stimuli are most similar in orientation but suppression is greatest when the orientations differ most. However, it has been previously found (Green, 1981a) that prolonged viewing of flicker or moving gratings raises threshold much more for test targets that are viewed with the same rather than the contralateral eye. Likewise, adaptation and testing with stationary gratings produces a much larger monoptic effect (e.g., Blakemore \& Campbell, 1969). It seems that the larger dichoptic effect depends on having both eyes stimulated simultaneously. This suggests that the larger dichoptic effect may, in fact, be due to some suppression mechanism.

\section{REFERENCES}

BAKer, C. (1963). Initial stages of dark and light adaptation. Journal of the Optical Society of America, 53, 98-103.

Blake, R., Breitmeyen, B., \& Green, M. (1980). Contrast sensitivity and binocular brightness: Dioptic and dichoptic conditions. Perception \& Psychophysics, 27, 180-181.

Blakemore, C., \& Campbell, F. W. (1969). On the existence of neurons in the human visual system selectively sensitive to orientation and size of retinal images. Journal of Physiology, 203, 237-260.

Boynton, R. M. (1961). Some temporal factors in vision. In W. A. Rosenblith (Ed.), Sensory communication. New York: Wiley.

Breitmeyer, B. G., \& Ganz, L. (1977). Temporal studies with flashed gratings: Inferences about human transient and sustained channels. Vision Research, 17, 861-866.

Campbell, F., \& Green, D. (1965). Monocular vs. binocular visual acuity. Nature, 208, 191-192.

Gilinsky, A., \& DoherTy, R. (1969). Interocular transfer of effects. Science, 164, 454-689.

Gore A, A. (1979). Directional and nondirection coding of a spatio temporal modulated stimulus. Vision Research, 19, 545-549.

Green, M. (1981a). Psychophysical relationships among mechanisms sensitive to pattern flicker and motion. Vision Research, 21, 971-984.

Green, M. (1981b). Spatial frequency effects in masking by light. Vision Research, 18, 861-866.

Green, M. (1983a). Detection and discrimination of drifting gratings masked by uniform flicker. Manuscript submitted for publication.

Green, M. (1983b). Masking by light and the sustained-transient dichotomy. Manuscript submitted for publication.

Green, M. (1983c). Visual masking by flickering surrounds. Vision Research, in press.

Green, M., \& Blake, R. (1981). Phase effects in monoptic and dichoptic temporal integration: Flicker and motion detection. Vision Research, 21, 365-372.

Kahneman, D. (1968). Method, findings and theory in studies of visual masking. Psychological Bulletin, 70, 404-425.

LEGGE, G. (1979). Spatial frequency masking in human vision: Binocular interactions. Journal of the Optical Society of America, 68, 838-847.

LEVELT, W. (1968). On binocular rivalry (Doctoral dissertation, Leiden University, 1965). The Hague: Mouton, 1968.

MAtin, L. (1962). Binocular summation at the absolute threshold of peripheral vision. Journal of the Optical Society of America, 52, 1276-1281.

Schiller, P. (1965). Monoptic and dichoptic visual masking by pattern and flashes. Journal of Experimental Psychology, 69, 193-199.

Sperling, G. (1965). Temporal and spatial masking. I. Masking by impulse flashes. Journal of the Optical Society of America, 55, 541-559.

Tolkurst, D. J. (1973). Separate channels for the analysis of shape and movement of a moving visual stimulus. Journal of Physiology, 231, 385-402.

TURvey, M. (1973). On peripheral and central processes in vision: Inference from an information-processing analysis of masking with patterned stimuli. Psychological Review, 80, 1-52.

WAgMan, I., \& Battersey, W. (1959). Neural limitations of visual excitability II: Retrochiasmal interaction. American Journal of Physiology, 197, 1237-1242.

Wetherill, G. B., \& LevitT, H. (1965). Sequential estimation of points on a psychometric function. British Journal of Mathematical and Statistical Psychology, 18, 1-9. 